\title{
LOS PADRES DEL NIÑO CON AUTISMO: UNA GUÍA DE INTERVENCIÓN
}

\section{M.Sc. Hannia Cabezas Pizarro}

Resumen: En este artículo se estudian las diferentes etapas por las que pasan los padres ante la llegada de un niño con autismo. Se presenta una definición de autismo, las principales características y los signos de alerta que podrían indicar que algo no marcha bien en el proceso de desarrollo de sus hijos.

Por otro lado, se definen algunos principios de la terapia conductual aplicada y se expone un diseño de un programa educativo para ayudar a los padres en el manejo de los niños con autismo. Partiendo del hecho de que los padres pueden ser coterapeutas de sus hijos, se les lleva a través de un proceso graduado en el que pueden elegir la conducta a trabajar y se les enseña cómo ordenar esas conductas de manera que se facilite la intervención.

Con este fin, se definen las conductas que presenta el niño autista y se indica cómo seleccionar los reforzadores o las consecuencias que éste recibirá, de modo que le permitan aumentar, mantener, o disminuir las conductas que se quieren cambiar; por lo que se ilustra cómo se miden éstas y se representan gráficamente, de forma que los padres puedan visualizar de manera sencilla los avances de sus hijos.

Palabras clave: AUTISMO/TERAPIA CONDUCTUAL/MANEJO DE NIÑOS/ CONDUCTAS/REFORZADORES/

\section{INTRODUCCIÓN}

Cuando un grupo familiar se enfrenta al nacimiento de un niño con autismo, los padres, hermanos (si los tiene) y parientes cercanos inician un camino difícil: el reto de entender las características que conforman este Síndrome, y unificarlas en torno al niño para poder manejarlo y educarlo adecuadamente.

\subsection{Definición}

El autismo es un trastorno generalizado del desarrollo, y se le llama trastorno autista. De acuerdo con el DSM-IV (APA, 1994, p. 65):

Se caracteriza por un déficit severo y generalizado en varias áreas del desarrollo: habilidades de interacción social recíproca, habilidades de comunicación, o por la presencia de conductas, intereses y actividades estereotipadas. El déficit cualitativo que define estos cuadros es distinto en su gravedad, en función del nivel de desarrollo o edad mental de los sujetos.

$\overline{\text { Bachiller en Retardo Mental de la Escuela de Orientación y }}$ Educación Especial de la Universidad de Costa Rica; Licenciada en Administración Educativa de la Universidad de Costa Rica; Maestría en Rehabilitación Integral de la Universidad de Costa Rica. Àreas de interés autismo. e-mail: hcabezas@cariari.ucr.ac.cr 
El trastorno autista se describe como una forma de los desórdenes profundos del desarrollo, caracterizados por:

a.- Un impedimento cualitativo de la interacción social recíproca.

b.- Un impedimento cualitativo en la comunicación verbal y no verbal en la actividad imaginaria.

c.- Una marcada restricción en el repertorio de actividades e intereses.

d.- El inicio en la primera infancia o en la niñez.

\subsection{El papel de los padres}

Los padres de un niño con autismo tienen que hacerle frente a una serie de dificultades, tanto en el campo emocional como en el eminentemente práctico. La presencia en la familia de un niño con alguna discapacidad se convierte en un factor potencial que puede perturbar, en forma significativa, la dinámica familiar. Una vez diagnosticado el trastorno, los padres han de pasar por un cambio de actitud, que implica un largo y doloroso proceso educativo, con un alto grado de estrés, que requerirá además de asesoramiento profesional cualificado.

Este tipo de trastorno les produce a los padres problemas emocionales característicos. Tomando en consideración los aspectos apuntados anteriormente, cuando el niño es pequeño (en general el diagnóstico no se realiza antes de 1-2 años de edad), los padres tienen que llegar a comprender las respuestas a interrogantes como las siguientes:

¿Por qué el niño llora constantemente sin una causa aparente?

¿Por qué no toma sus alimentos en la misma forma que los niños de su misma edad?

¿Por qué presenta conductas repetitivas?

¿Por qué no acepta el contacto físico o permanece aislado la mayor parte del tiempo? y, ¿por qué no sabe comunicarse, ni utilizar el lenguaje en forma apropiada?

El papel de los padres es de suma importancia en la integración social del niño, sólo así se dará una mejor convivencia que permita mantener a la familia unida. Esto por cuanto las conductas que presentan los niños con autismo son un factor disruptivo en el entorno, y muchas veces constituyen la causa de la desintegración de los núcleos familiares. El comportamiento del niño, por sí solo, influye el ambiente. Un niño que llora, que corre de un lado a otro arrasando con lo que encuentra, que hace berrinches sin una causa aparente en forma sistemática y sostenida y que se aísla y presenta conductas repetitivas, se convierte en un factor que desencadena estrés y en una fuente de desequilibrio para la armonía del hogar. 


\subsection{Proceso educativo para el niño y su familia}

La base del proceso educativo para los niños con autismo, se sustenta en la detección temprana, y en la puesta en marcha de programas de intervención conductual. El tratamiento conductual implica la aplicación sistemática de los principios del aprendizaje a la conducta humana: los principios derivados del análisis experimental de la conducta (ciencia que tiene por objetivo identificar y comprender las leyes por las que los factores ambientales rigen la conducta). Una vez que se han identificado, esas leyes se usan para cambiar la conducta de los individuos; práctica que se denomina como modificación de conducta. En este proceso juega un papel importante la información que se les brinde a los padres, el apoyo psicológico profesional y la referencia de los servicios disponibles en la comunidad para el tratamiento de su hijo y el apoyo a la familia.

El trabajo es arduo. Los padres se enfrentan a la pérdida inicial del niño "ideal" que habían conformado en su mente. Esa imagen se distorsiona por la imagen real, lo que desencadena una serie de sentimientos como negación, dolor, culpa, frustración, cólera y llanto, hasta que se acepte el problema.

\subsubsection{Etapas que viven los padres}

La etapa de negación se caracteriza por el aturdimiento y por la incredulidad (que eso les esté pasando a ellos no es posible), lo que manifiestan con la pregunta: “¿por qué a mí?”. Aquí se inicia un largo peregrinar por diferentes profesionales, con la esperanza de que alguno les diga que hay una equivocación en el diagnóstico inicial.

En la mayoría de los casos, el término autismo por sí solo no significa nada para los padres, puesto que ellos no poseen conocimientos acerca del síndrome ni de sus proyecciones futuras. La desinformación, unida al sentimiento de duelo que el padre y la madre no entienden, los llevan a experimentar cólera por la pérdida del "niño ideal" sobre el cual se habían cifrado un sin número de esperanzas.

Otro sentimiento que aparece es el de miedo. Principalmente, si los padres no tienen una orientación profesional adecuada, o si el niño no tiene una intervención temprana, se generan sentimientos de temor hacia un futuro para el que no tienen respuesta... ¿Ahora qué pasará? Preguntas como: qué dirán, cómo lo manejarán con los otros hermanos, o con los parientes, o si afectará o no la relación de pareja, no se hacen esperar.

Adicionalmente, aparece la incertidumbre acerca de si los ambientes educativos a los que asistirá el niño o los maestros que lo atenderán, estarán preparados para su educación. 
La culpa aflora en los padres porque creen que son la causa del problema y empiezan a indagar en la historia familiar buscando una respuesta que no encuentran.

El rechazo hacia el niño y a todo aquello que los rodea, sumado a sentimientos de tristeza, dolor y vergüenza, entre otros, hace que en muchos casos, como una forma de evadir el problema, aparezcan en los padres la depresión o los deseos de morir. Estos sentimientos son normales, parte del proceso de ajuste que enfrenta el ser humano ante situaciones dolorosas.

Durante la sucesión de estas etapas son necesarios los grupos de apoyo que ayudan a satisfacer las necesidades de los padres; de modo que les permitan entender los sucesos racionalmente y así poder ayudar en forma efectiva a sus hijos y a los otros miembros del grupo familiar, mientras empiezan a comprender mejor los ajustes que hay que realizar.

\subsubsection{Algunas anotaciones acerca de los problemas a los que se enfrentarán los padres}

Se ha mencionado (Orjales y Polaino, 1993) que en aquellos hogares donde vive un niño con autismo se presentan más problemas familiares que en aquellas familias en las que los hijos no tienen necesidades educativas especiales; sin embargo, este no es un factor determinante para que se altere el orden familiar, sino que hay otros aspectos asociados que inciden en ello, como la estabilidad emocional de cada uno de los miembros del núcleo familiar, la educación de los padres y, sobre todo, el proceso de aceptación del niño.

Las barreras sociales son otro impedimento al que se enfrentan los progenitores. El rechazo por parte de amigos, maestros y directores de centros educativos, que por el temor que se les genera su desconocimiento sobre lo que tienen que hacer o sobre cómo guiarlos en el quehacer educativo; aumentan la inseguridad que viven los padres.

Cuxart (1997) y Wing (1998) citan algunas de las reacciones más frecuentes en las parejas ante la llegada de un niño con necesidades especiales, y argumentan que la discapacidad de una persona dentro del seno familiar, puede fortalecer o debilitar a la pareja. Estos investigadores, también toman en cuenta otros factores que inciden en crisis, como el nivel cultural y económico, la estabilidad emocional de los padres, la presencia o no de más hijos y la severidad de la discapacidad. En relación con el nivel de severidad, Cuxart (1996, 1997 ) realizó estudios que concluyeron que a mayor grado de severidad de los problemas en los niños, mayor es el nivel de estrés generado en los padres.

Esto nos lleva a pensar que si los padres reciben apoyo profesional en forma de entrenamiento, de modo que les permita manejar mejor las conductas de sus hijos, los niveles 
de estrés bajarán considerablemente y esto les ayudará a convivir con su problema. Por lo tanto, se hace necesario contar con programas muy bien estructurados (bajo la guía de profesionales en este campo) que les dé a los padres de familia la oportunidad de aprender las destrezas básicas que les permitan tener una mejor interacción con sus hijos y con los otros miembros del grupo familiar y social.

\subsubsection{Algunos rasgos característicos de la conducta autista}

Como una medida para un diagnóstico y una intervención temprana los padres deben estar atentos a una serie de signos que presentan los niños con autismo:

1. Si llora sin una causa aparente.

2. Si no tiene lenguaje.

3. Si no establece contacto ocular.

4. Si le gusta permanecer solo.

5. Si rechaza el pecho materno.

6. Si hace berrinche sin una causa aparente.

7. Si al hablarle pareciera que no escucha.

8. Si no tiene noción de peligro.

9. Si presenta movimientos repetitivos.

10. Si rechaza el contacto físico.

La presencia de algunas de las conductas de los niños con autismo son generadas por la incapacidad de éste para entender qué es lo que está sucediendo a su alrededor, y el llanto incontrolado, los gritos y los berrinches, se convierten en su forma de comunicación. Su conducta es la manera de decirnos que algo está pasando.

Todos los niños tienen posibilidades de aprender, y los padres también pueden convertirse en maestros de sus propios hijos.

Los programas que pongan en ejecución los educadores especializados deben estar muy bien estructurados y sistematizados, ya que de ello dependerán los resultados que se obtengan.

Cada persona con autismo es diferente, por lo que los programas deben ser individualizados y dirigirse a las necesidades particulares de cada una. 


\section{TERAPIA CONDUCTUAL APLICADA}

Las investigaciones realizadas (Lovaas, 1981; Rivière, 1997a, b; Cuxart; 1997) indican que lo que mejores resultados ha dado en el trabajo con personas con autismo, ha sido el uso de la terapia conductual aplicada. Es por ello que en este artículo se desarrolla un programa basado en la utilización de esta técnica.

\subsection{Principios acerca de Terapia Conductual}

Como se mencionó anteriormente, el tratamiento conductual aplicado es un método altamente sistematizado de modificación de la conducta. Se usa principalmente para el mejoramiento de problemas conductuales y en programas para el aprendizaje. La terapia conductual está basada en el rendimiento, ya que los cambios en las conductas concretas son evaluados objetivamente mediante un método analítico que emplea mediciones directas y repetidas experimentalmente. De esa forma, se puede evaluar continuamente si un procedimiento que se está aplicando es o no efectivo.

Al aplicar un tratamiento conductual, el uso de reforzadores es de suma importancia. Cuando se presenta un estímulo, o este aparece como consecuencia de o dependiente de una respuesta, y cuando el porcentaje de esa respuesta aumenta, se dice que hay un reforzamiento positivo. Por otra parte, el reforzamiento negativo implica la eliminación de un estímulo aversivo. Cuando se aplica un castigo, se está ante una consecuencia aversiva. La extinción es el término utilizado para indicar que el reforzamiento se ha dejado de presentar.

Desde hace muchos años, los psicólogos (Pavlov, 1960; Watson y Raynor, 1920; Wolf, Risley y Mees, 1964) descubrieron tres cosas importantes que se han convertido en los postulados que sustentan la terapia conductual aplicada:

-Una persona aprende a hacer algo a cambio de lo que obtiene una recompensa.

-Una persona deja de hacer algo a cambio de que no obtiene ninguna recompensa.

-Una persona aprende mejor si le enseñamos a partir de pequeños pasos.

De lo anterior se desprende que si reforzamos en forma adecuada aquellas conductas que queremos incrementar, la posibilidad de que vuelvan a ocurrir aumenta. En la misma medida, si eliminamos los reforzadores que están manteniendo las conductas que queremos eliminar, estas tienden a desaparecer.

Dependiendo de cómo manejemos estos procedimientos, podríamos hacer que las conductas con las que queremos trabajar aumenten, disminuyan o se mantengan.

Ante la presencia de un estímulo (que puede ser auditivo, táctil, visual, gustativo, olfativo, etc.) aparece una respuesta, y esta respuesta se incrementa, se mantiene o disminuye, de 
acuerdo con las consecuencias que reciba. Estas consecuencias pueden ser agradables o desagradables.

A esta triada de elementos que interactúan entre sí conformando los comportamientos, se le ha denominado "relación de triple contingencia", y se representa con las letras:

$E \rightarrow R \rightarrow C$, o (estímulo, respuesta, consecuencia)

Como ejemplo podríamos citar el siguiente:

Manuel va al supermercado con su madre, pasa por el estante de los helados. Le pide a su madre que le compre uno, a lo que ella se niega. Ante esta respuesta, Manuel llora en forma desproporcionada y empieza a llamar la atención de los clientes que se encuentran allí. Su madre, con tal de que Manuel deje de llorar, le compra los helados.

\section{Identificando las situaciones tenemos:}
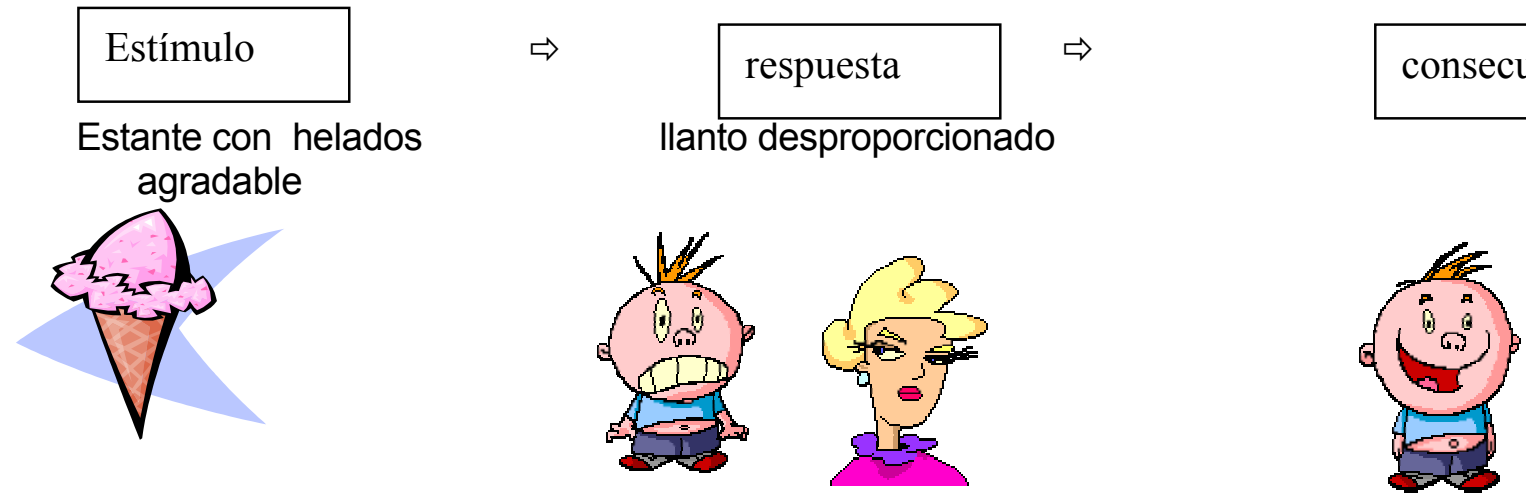

En este caso, la probabilidad de que Manuel llore en forma desproporcionada cuando pase de nuevo por el estante de los helados va a aumentar, ya que la conducta de llorar se reforzó.

Este es un claro ejemplo de cómo aprenden las personas. En ocasiones, las conductas se incrementan en forma accidental o por desconocimiento de los procesos que intervienen cuando se establece relaciones entre las conductas y las consecuencias que se dan.

¿Qué tipo de consecuencias podríamos proporcionar?

Como parte de estas consecuencias tenemos los llamados reforzadores. Pueden ser primarios, es decir, aquellos que tienen carácter concreto y satisfacen necesidades biológicas, como las cosas comestibles, el abrigo y el sexo. 
También existen reforzadores sociales, resultado de la interacción social, como por ejemplo los abrazos, las alabanzas, la aprobación, y las sonrisas.

Existen además, los reforzadores de actividad, que consisten en permitirle al niño realizar alguna actividad de su agrado, como: salir de paseo, ver televisión, ir al cine.

Por su parte, el castigo tipo I es una consecuencia en la que no hay contacto directo con el niño, y consiste, por ejemplo, en retirarlo del sitio donde se encuentre o sentarlo por un tiempo determinado en un lugar específico.

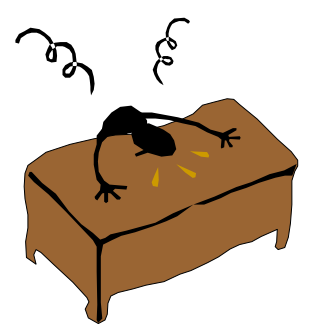

El castigo tipo II conlleva el contacto físico directo con el niño, como una palmada.

Otro principio conductual que se aplica extensivamente, es el moldeamiento; éste consiste en la división de una conducta en sus componentes; aquí se refuerza cada aproximación a la conducta final deseada. (Buckely y Walker, 1970).

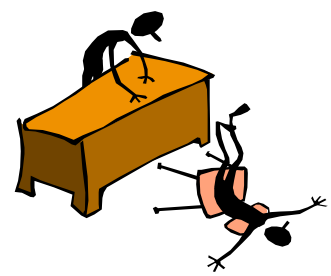

Como ejemplo, supongamos que un niño no permanece sentado.

Para aplicar el procedimiento, lo primero que se hace es reforzar cualquier intento del niño de acercarse a la silla; luego, se le refuerza cuando se acerca a determinada distancia y no se le refuerza si se aleja de la silla. Posteriormente se le refuerza si el niño permanece alrededor de la silla aunque no se siente, $y$, el paso siguiente, será reforzar al niño cuando se siente en la silla. Progresivamente se intentará que el niño permanezca sentado por más tiempo, hasta llegar al criterio final previamente definido para la conducta que se desea alcanzar.

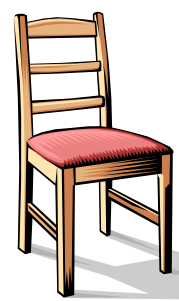

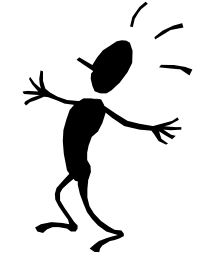

Reforzar

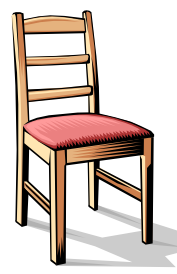

Reforzar
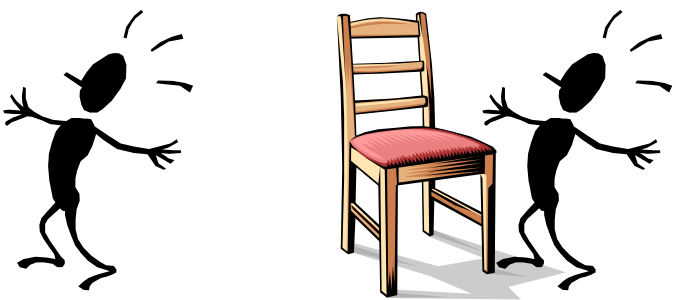

Reforzar 
Otra consecuencia es la extinción, que consiste en "suspender la entrega de reforzamiento, es decir, suprimir consecuencias que siguen a determinada conducta" (Buckely y Walker, 1970, p.128).

Poner en extinción una conducta significa no prestarle atención o ignorarla cada vez que ésta se presente.

\subsection{Consejos para los padres al reforzar a sus hijos}

Al poner a punto un programa educativo, Becker (1989, p. 39) les recomienda a los padres tomar en consideración las siguientes tres reglas para reforzar a sus hijos:

1. Cuando se trate de enseñarles tareas nuevas, se reforzará inmediatamente sin permitir que haya un retraso entre la respuesta y el reforzamiento.

2. En las etapas iniciales del aprendizaje de una tarea, debe reforzarse cada emisión.

3. Es necesario reforzar el mejoramiento o los avances en la dirección correcta. No hay que insistir en una ejecución perfecta desde el primer ensayo.

Recordemos que los padres son maestros de sus hijos y que tienen que jugar un rol de corresponsabilidad en algunas de las áreas de intervención mediante una programación conjunta con el terapeuta. Es muy importante que se tenga un plan lo más estructurado posible para el hogar.

A continuación se desarrolla una guía práctica que le permitirá a los padres tener en el hogar y en el entorno social, un mejor control de las conductas disruptivas de sus hijos.

\section{DISEÑO DE UN PROGRAMA EDUCATIVO INDIVIDUAL}

\subsection{Primer paso: Eligiendo una conducta.}

Al trabajar con niños con autismo, nos preguntamos:

¿Qué es lo que necesitan aprender?

¿Por dónde empezar?

¿Qué es lo primero que debemos enseñarles?

Blackman y Silberman, (1973, p.15) establecieron criterios que se deben considerar a la hora de cambiar una conducta, entre ellos están:

1. La conducta a cambiar debe presentarse con cierta frecuencia

2. Si la conducta afecta al niño y su entorno

3. La conducta impide una evolución saludable 
Por otra parte, si las conductas se presentan en períodos espaciados, o si estas desaparecen con el tiempo, no son susceptibles de intervención, ya que forman parte del desarrollo normal de los niños.

Las conductas, para ser intervenidas, no deben elegirse en forma arbitraria, porque eso solo dificultaría el proceso. Se debe tener en consideración que unas conductas son prerrequisito de otras, para que sobre ellas, se estructure el aprendizaje.

Como primer paso para la selección de las conductas a intervenir, debe elaborarse un registro de observación, que consista en ir anotando todas las cosas que el niño hace en un tiempo determinado. Esta labor permite tener una visión global del comportamiento del niño en un ambiente específico. Se puede realizar en el hogar, la escuela, o un lugar público. Esto hace que deban delimitarse, con anticipación, las condiciones en las que se harán las observaciones y su control.

\section{Ejemplo:}

María es una niña que llora constantemente, corre de un lugar a otro en su casa y no permite que se le acerquen. No permanece sentada. En ocasiones, se balancea sobre su propio cuerpo, se le habla y sigue caminando como si no hubiese escuchado, además, no establece contacto visual.

\section{Primer paso:}

\section{Seleccionando las conductas de María}
a. Llora,
b. no permanece sentada,
c. se balancea,
d. no acepta el contacto físico,
e. no establece contacto visual,
f. no sigue instrucciones.

\subsection{Segundo paso: Ordenando las conductas}

Para un mejor rendimiento del niño, debe establecerse una jerarquía de las conductas de acuerdo con el grado de dificultad o si una necesita ser antecedida de otra.

Por ejemplo: 
Si se quieren trabajar varios aspectos, entre ellos que el niño llora, el proceso es más lento si se inicia por tratar de controlar el llanto, ya que el niño no permanece quieto, no sigue instrucciones y no mantiene el contacto ocular.

El orden lógico sería: En primer lugar, sentarlo. En segundo lugar, controlar el llanto; luego, enseñarlo a establecer contacto ocular adecuado, y por último, enseñarlo a seguir instrucciones.

Hay conductas que se pueden trabajar simultáneamente, como permanecer sentado y el controlar el llanto.

Es necesario, una vez seleccionada la conducta, definirla, lo que permitirá saber en qué momento y en que forma debe ser intervenida.

\subsection{Tercer paso: Definiendo las conductas}

Las conductas deben ser definidas en términos observables y medibles. ¿Qué significa esto? Que se puedan contar las veces que ocurre una conducta determinada en un intervalo de tiempo específico.

Por ejemplo, utilizando un caso en el que se requiere control del llanto, se puede definir la conducta de la siguiente manera:

Llanto: Gritos emitidos por el niño, lo suficientemente altos, que se oyen a una distancia de 10 a 15 metros, y cuya duración es mayor de 5 minutos.

Puedo determinar cuántas veces lo hace el niño al día: 10, 20,...etc.

¿Cuánto dura cada período? 10 minutos, 15 minutos,...etc.

\subsection{Cuarto paso: Seleccionando los reforzadores}

Antes de seleccionar un reforzador que surta efecto en el niño, es necesario hacer un muestreo de reforzadores, de tal manera que sepamos qué es importante y qué no es importante para el niño. Para ello, Blackman y Silverman (1973, p. 44) apuntan que..."un estímulo no posee ninguna propiedad reforzadora inherente. Tenemos que determinarla 0 descubrirla (es decir, debemos llegar a conocer las capacidad que tiene el estímulo de acrecentar la probabilidad de la respuesta)."

¿Qué debo hacer?

-Elaborar una lista de las cosas que son importantes para el niño. Por ejemplo, productos comestibles como galletas, jugos, chocolates, etc., juguetes diversos, o actividades como ver televisión y jugar bola, o actividades recreativas como ir a algún lugar específico. Esta lista será utilizada para reforzar aquellas conductas que queremos cambiar. 
¿Cómo funciona este proceso?

Pavlov (1960) fue el primero en estudiar el condicionamiento clásico. Trabajando con un perro, él descubrió que si hacía sonar una campanilla y acompañaba este sonido con un poco de comida, el perro salivaba. Realizó muchas veces este procedimiento, y al cabo del tiempo descubrió que el solo sonido de la campanilla provocaba la salivación del perro. Posteriormente, mediante la experimentación, demostró que el uso de un estímulo neutro (campanilla), que en un inicio por sí solo no tenía ningún significado, si se asociaba a un estímulo no condicionado (la comida), podía conseguir una respuesta (salivación) por parte del perro. El condicionamiento Pavloriano nos indica cómo al combinar un estímulo condicionado con otro incondicionado, a través de la repetición, puede obtener resultados positivos en las conductas que se están trabajando. Uno de los descubrimientos importantes de Pavlov fue el proceso de extinción. Observó que si el estímulo no condicionado se omitía en una serie de pruebas de condicionamiento, la respuesta condicionada empezaba a disminuir y finalmente desaparecía (Blackman y Silverman, 1973, p. 37-38).

\subsection{Quinto paso: Midiendo la conducta}

Para saber si hay progresos cuando se ha intervenido una conducta, es necesario medirla. Para ello, podemos usar varios criterios como la intensidad y la frecuencia con que se presenta. Es necesario que se observe conducta y se anote el número de veces que aparece.

Por ejemplo, si Mario grita constantemente, podemos anotar el número de veces que esta conducta se da durante un período, por ejemplo, una semana.

Es necesario diseñar una hoja que nos permita anotar la frecuencia con que se presenta la conducta.

Tabla 1

Control de llanto

\begin{tabular}{|l|l|l|l|l|l|l|l|}
\hline Días & lunes & martes & miércoles & jueves & viernes & sábado & domingo \\
\hline Veces & 9 & 10 & 9 & 11 & 14 & 10 & 11 \\
\hline Tiempo & $15 \mathrm{~min}$ & $12 \mathrm{~min}$ & $16 \mathrm{~min}$ & $11 \mathrm{~min}$ & $10 \mathrm{~min}$ & $12 \mathrm{~min}$ & $15 \mathrm{~min}$ \\
\hline
\end{tabular}

En el registro anterior, podemos observar el número de veces que Mario gritó durante los días de una semana, lo que nos da un promedio aproximado de 11 veces al día. 


\subsection{Sexto paso: Graficando la conducta}

El gráfico nos permite visualizar la conducta, y si ésta disminuye o aumenta al aplicar los procedimientos conductuales elegidos para la intervención.

Gráfico de conducta control de llanto, antes de la intervención.

\section{Grafico 1}

Control de llanto

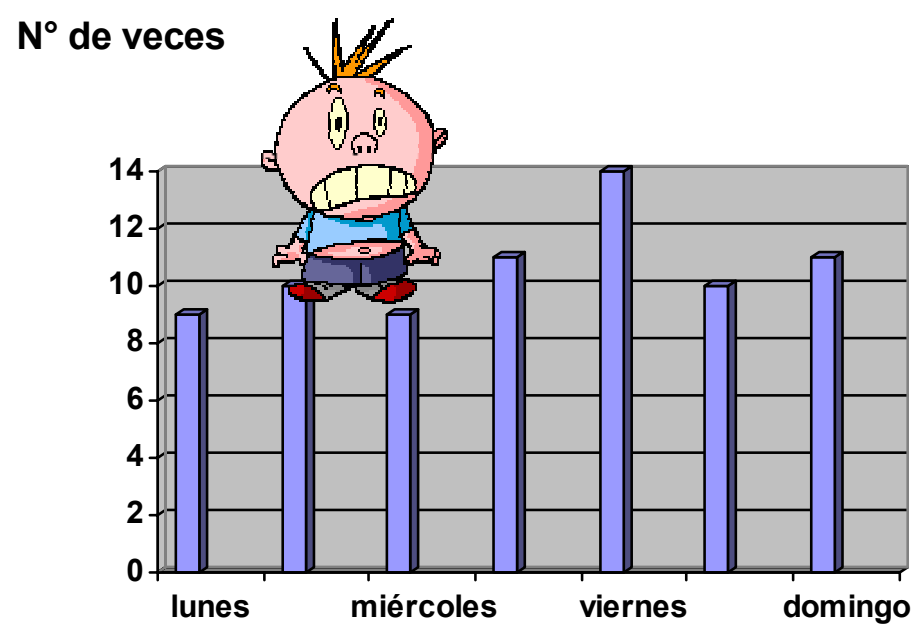

días de la semana

En el eje vertical se representa el número de veces en que Mario lloró, y en el horizontal los días de la semana.

\subsection{Sétimo paso: Escogiendo los procedimientos para la intervención}

En este paso se seleccionarán los procedimientos que se aplicarán y los reforzadores, ya sean productos comestibles o actividades que le gusten al niño.

La madre de Mario decidió reforzar con helados los períodos en que este permanecía sin llorar, asociando este reforzador con uno social, al decirle, cada vez que le daba helados, “¡Qué bien que estás sin llorar!" A su vez, utilizó otro procedimiento conductual (aislamiento) y lo aplicó cada vez que Mario lloraba y gritaba, retirándolo a su habitación. De ese modo, se obtuvieron los resultados anotados en la Tabla 2 después de registrar su conducta durante siete días. 
Tabla 2

Control de llanto

\begin{tabular}{|l|l|l|l|l|l|l|l|}
\hline Días & lunes & martes & miércoles & jueves & viernes & Sábado & domingo \\
\hline Veces & 15 & 12 & 16 & 8 & 6 & 5 & 4 \\
\hline Duración & $16 \mathrm{~min}$ & $14 \mathrm{~min}$ & $15 \mathrm{~min}$ & $10 \mathrm{~min}$ & $5 \mathrm{~min}$ & $3 \mathrm{~min}$ & $3 \mathrm{~min}$ \\
\hline
\end{tabular}

Una vez que se aplicaron los procedimientos conductuales, la conducta de llorar empezó a decrecer, lo que se observa en la Tabla 2.

El Gráfico 2, nos da una idea visual de cómo fue incrementándose la conducta de Mario de permanecer sin llorar.

\section{$\mathbf{N}^{\circ}$ de veces}

\section{Gráfico 2}

Control de Ilanto

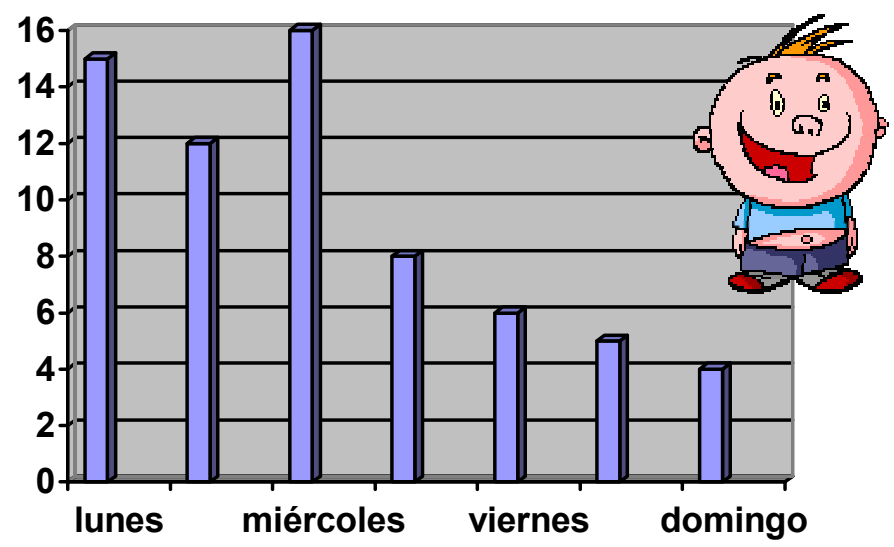

días de la semana

En el eje vertical se indica el número de veces que se presenta la conducta de llorar y en el horizontal, los días de la semana. Como se observa en la gráfica, en los días iniciales se da un incremento de la conducta, proceso que es normal, puesto que la madre de Mario comenzó a ignorar los berrinches. Conforme se aplicaron los procedimientos conductuales (en este caso se reforzó la conducta de permanecer en silencio), el llanto casi desapareció.

Esta guía, muestra uno de los procedimientos conductuales que pueden aplicar los padres para mejorar las conductas de sus hijos. Dentro de la terapia conductual, existen muy variadas intervenciones, las cuales, pueden aprenderse fácilmente. 


\section{CONCLUSIÓN}

Durante los últimos años en mi experiencia con padres y madres, he comprobado que estos desean ser coterapeutas en la educación de sus hijos, por lo que es necesario involucrarlos en el proceso educativo; su presencia es importante en las sesiones de terapéutica. Esto les permitirá, con la ayuda del educador, incorporar y poner en práctica los procedimientos conductuales y contribuir eficazmente en la educación de sus hijos.

El conocimiento de las diferentes técnicas y la puesta en práctica de las mismas, les brindará a los padres una mejor comprensión del síndrome y un mejor control del estrés como consecuencia, al ver los progresos de sus hijos. Además, las relaciones e interacciones familiares mejoran, ya que la convivencia con un niño que presente las características de los niños con autismo es muy particular; esto por la falta de contacto afectivo, la ausencia de comunicación verbal y la presencia de conductas peculiares características de este síndrome.

\section{REFERENCIAS}

American Psychiatric Association. (1994). Diagnostic and Statistical Manual of Mental Disorders. Fourth Edition, DSM-IV. Washington D.C.: A.P.A.

Blackman, G. y Silberman, A. (1973). Como modificar la conducta infantil. Buenos Aires: Editorial Kapelusz.

Becker, W.C. (1989). Los padres son maestros. Programa de manejo infantil. México: Editorial Trillas.

Buckely, N.K. y Walker, H.M. (1970). Modificación de conducta en el salón de clase. Manual de procedimientos para el maestro. México: Instituto Interamericano de Estudios Psicológicos y Sociales.

Curxart, F. (1996). Los autistas severamente afectados. Problemas conductuales, Estrés familiar y tratamientos continuados. Ponencia presentada en el $5^{\circ}$ Congreso AutismoEuropa, Barcelona.

Cuxart, F, (1997). La familia del niño con autismo y el apoyo terapéutico a las familias. En: Rivière, A. y Martos, J. (Comp.) El tratamiento del autismo. Nuevas perspectivas. Madrid: Ministerio de Trabajo y Asuntos Sociales.

Lovaas, O.I. (1981). El niño autista. Madrid: Editorial Debate.

Orjales, I. y Polaino, A. (1993). Impacto y consecuencias psicopatológicas del retraso mental en la familia. Revista Complutense de Educación, 4 (2), 67-69.

Pavlov, I.P. (1960). Conditioned reflexes. New York: Dover Publications. 
Rivière, A. (1997a). El tratamiento del autismo como trastorno del desarrollo: principios generales. En: Rivière, A. y Martos, J. (Comp.) El tratamiento del autismo. Nuevas perspectivas. Madrid: Ministerio de Trabajo y Asuntos Sociales.

Rivière, A. (1997b). Modificación de conducta en el autismo infantil. En: Rivière, A. y Martos, J. (Comp.) El tratamiento del autismo. Nuevas perspectivas. Madrid: Ministerio de Trabajo y Asuntos Sociales.

Watson, J.B. y Raynor, R. (1920). Conditioned emotional reactions. Journal of Experimental Psychology, 3, 1-14.

Wing, L. (1998). El autismo en niños y adultos. Una guía para la familia. Barcelona: Editorial Piados Ibérica.

Wolf, M., Risley, T., Mees, H. (1964). Aplication of operant conditioning procedures to the behavior problems of an autistc child. Behaviour Research and Therapy 1, 305-312.

\section{BIBLIOGRAFÍA ADICIONAL}

Baron-Cohen, S. y Bolton, P. (1998). Autismo, Una guía para padres. Madrid: Editorial Alianza.

Harppé, F. (1998). Introducción al autismo. Madrid: Editorial Alianza.

Harris, S. (2001). Los hermanos de los niños con autismo. Madrid: Ediciones Narcea.

Hobson, R.P. (1995). El autismo y el desarrollo de la mente. Madrid: Editorial Alianza.

Krumboltz, J.D. y Krumboltz, H.B. (1976). Como Cambiar la conducta del niño. Buenos Aires: Editorial Guadalupe.

Martos, J. (1984). Los padres también educan. Guía Práctica. Madrid: APNA.

Martos, J. y Rivière, A. (Comp.). (2001). Autismo: compresión y explicación actual. Madrid: Ministerio de Trabajo y Asuntos Sociales.

Polaino, A., Doménech, E. y Cuxart, F. (1997). El impacto del niño autista en la familia. Instituto de Ciencias para la familia. Universidad de Navarra, España: Ediciones Rialp. 